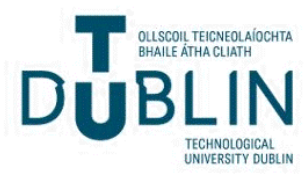

Technological University Dublin ARROW@TU Dublin

2015-5

\section{On Mikhailov's Reduction Group}

\author{
Tihomir Valchev \\ Technological University Dublin, Tihomir.Valchev@tudublin.ie
}

Follow this and additional works at: https://arrow.tudublin.ie/scschmatart

Part of the Mathematics Commons, and the Partial Differential Equations Commons

\section{Recommended Citation}

T. Valchev, On Mikhailov's reduction group, Physics Letters - A 379 (2015) 1877-1880. doi:10.1016/ j.physleta.2015.05.024

This Article is brought to you for free and open access by the School of Mathematics at ARROW@TU Dublin. It has been accepted for inclusion in Articles by an authorized administrator of ARROW@TU Dublin. For more information, please contact arrow.admin@tudublin.ie, aisling.coyne@tudublin.ie, gerard.connolly@tudublin.ie. Funder: Government of Ireland Postdoctoral Fellowship in Science, Engineering and Technology 


\title{
On Mikhailov's Reduction Group
}

\author{
Tihomir Valchev*† \\ School of Mathematical Sciences, \\ Dublin Institute of Technology, \\ Kevin Street, Dublin 8, Ireland
}

\begin{abstract}
We present a generalization of the notion of reduction group which allows one to study in a uniform way certain classes of nonlocal $S$-integrable equations like Ablowitz-Musslimani's nonlocal Schrödinger equation. Another aspect of the proposed generalization is the possibility to derive in a systematic way solutions to $S$-integrable equations with prescribed symmetries.
\end{abstract}

\section{Introduction}

Symmetries play a quite significant role in mathematics and physics. This is especially true for contemporary mathematical physics where notion of integrability is of fundamental importance. Although there are various definitions of integrability [12] they all are manifestation of certain underlying symmetries.

Our paper is dedicated to a very special type of symmetries known as reductions of integrable nonlinear equation (NLE). Those are discrete symmetries of auxiliary spectral problem related to NLE which are a central topic in theory of integrable systems $[5,9,13]$. We aim here to propose an extension of the original Mikhailov's notion of reduction group [8] by allowing transformation of independent variables $x$ and $t$. Despite being quite simple, such possibility seems to have been neglected ever since the reduction group was invented. It is our intention here to show that idea deserves serious consideration. There are at least two reasons for this. First, the afore-mentioned generalization would allow for new classes of NLEs to be treated through the inverse scattering method (ISM). A representative example of such equation is nonlocal nonlinear Schrödinger equation (NNS)

$$
\mathrm{i} q_{t}+q_{x x} \pm 2 q^{2}(x, t) q^{*}(-x, t)=0, \quad q: \mathbb{R}^{2} \rightarrow \mathbb{C}
$$

where $*$ means complex conjugation. NNS was proposed by Ablowitz and Musslimani [1] as a model for wave propagation in $P T$-symmetric wave guides.

Another important advantage of including the independent variables into reduction group action is that it yields to a systematic approach to generate

\footnotetext{
*E-mail address: Tihomir.Valchev@dit.ie .

† On leave from the Institute for Nuclear Research and Nuclear Energy, Bulgarian Academy of Sciences, 72 Tsarigradsko chausse, Sofia 1784, Bulgaria.
} 
solutions to local NLEs with certain prescribed symmetries. It is already known that such solutions could find rather nontrivial applications. For example, it has been shown by Wadati [11] that solutions to the classical mKdV

$$
u_{t}+u_{x x x}+6 u^{2} u_{x}=0, \quad u: \mathbb{R}^{2} \rightarrow \mathbb{R}
$$

with the symmetry $u(-x, 0)=u(x, 0)$ imposed can be used to construct nonHermitian quantum mechanical Hamiltonians whose spectrum is real.

Our paper is organized as follows. Section 2 is preliminary in nature. Its purpose is to introduce some basic notions from the direct scattering problem for polynomial bundle Lax operators which will be used further in text. In next section we present our main results. We discuss the concept of reductions as it is understood in theory of integrable systems and demonstrate how it could be generalized. Several examples to illustrate the new concept of reduction will be given. Finally in Section 4 we summarize our results and make some additional remarks.

\section{General Remarks on Polynomial Bundles}

In this section we shall briefly sketch the foundations of the direct scattering problem for generic (that is non-reduced) polynomial bundles. Our exposition will be mostly based upon conventions used in $[3,4,5,7]$.

Let us consider Lax operators

$$
\begin{array}{lll}
L(\lambda)=\mathrm{i} \partial_{x}+U(x, t, \lambda), & & U: \mathbb{R}^{2} \rightarrow \mathfrak{g}[\lambda], \\
A(\lambda)=\mathrm{i} \partial_{t}+V(x, t, \lambda), & V: \mathbb{R}^{2} \rightarrow \mathfrak{g}[\lambda]
\end{array}
$$

where subscripts stand for differentiation in variables $x$ and $t$ and $\mathfrak{g}[\lambda]$ denotes Lie algebra of polynomials in spectral parameter $\lambda \in \mathbb{C}$ with coefficients in a complex simple Lie algebra ${ }^{1} \mathfrak{g}$. The compatibility condition (zero curvature condition)

$$
[L(\lambda), A(\lambda)]=0
$$

of Lax operators can be rewritten in terms of functions $U$ and $V$ as follows:

$$
U_{t}-V_{x}+\mathrm{i}[U, V]=0 .
$$

Making use of root space decomposition [6] we can represent each matrix coefficient of the polynomial

$$
U(x, t, \lambda)=\sum_{k=0}^{M} U_{k}(x, t) \lambda^{k}
$$

in the form:

$$
U_{k}=U_{k}^{\mathrm{n}}+U_{k}^{\mathrm{d}}, \quad U_{k}^{\mathrm{d}} \in \mathfrak{h}, \quad U_{k}^{\mathrm{n}} \in \sum_{\alpha \in \Delta} \mathfrak{g}_{\alpha}
$$

where $\mathfrak{h} \subset \mathfrak{g}$ is the Cartan subalgebra of $\mathfrak{g}, \Delta$ is the root space of $\mathfrak{g}$ and $\mathfrak{g}_{\alpha} \subset \mathfrak{g}$ is the root space corresponding to root $\alpha$. Following [7] we shall restrict ourselves with polynomial bundles for which

$$
U_{k}^{\mathrm{d}}=\text { const }, \quad U_{M}^{\mathrm{n}}(x, t) \equiv 0, \quad U_{M}^{\mathrm{d}} \neq 0 .
$$

\footnotetext{
${ }^{1}$ We shall be interested in matrix Lie algebras.
} 
Moreover, we shall require that all dynamical fields vanish sufficiently fast when $x \rightarrow \pm \infty$ so that $U$ obeys boundary condition

$$
\lim _{|x| \rightarrow \infty} U(x, t, \lambda)=-\mathcal{J}(\lambda) \in \mathfrak{h} .
$$

Let us consider the following examples.

Example 1 Assume that the Lax operator $L(\lambda)$ is linear in the spectral parameter. Then it can be written down in the form:

$$
L(\lambda)=\mathrm{i} \partial_{x}+Q(x, t)-\lambda J .
$$

Above $J \in \mathfrak{h}$ is a constant matrix while

$$
Q(x, t)=\sum_{\alpha \in \Delta} Q_{\alpha}(x, t) E_{\alpha}
$$

where $E_{\alpha}$ denotes the Weyl generator related to root $\alpha$. In this particular case the boundary condition (9) translates into

$$
\lim _{|x| \rightarrow \infty} Q(x, t)=0 .
$$

Example 2 Suppose now that $L(\lambda)$ is a quadratic polynomial in $\lambda$ of the following form:

$$
L(\lambda)=\mathrm{i} \partial_{x}+Q_{0}(x, t)+\lambda Q_{1}(x, t)-\lambda^{2} J .
$$

Like in the previous example $J \in \mathfrak{h}$ is a constant matrix and $Q_{1}(x, t)$ is a linear combination of Weyl generators of $\mathfrak{g}$. The $\lambda$-free term is arbitrary element of $\mathfrak{g}$, i.e. it can be decomposed in the following manner:

$$
Q_{0}(x, t)=Q_{0}^{\mathrm{n}}+Q_{0}^{\mathrm{d}}, \quad Q_{0}^{\mathrm{d}} \in \mathfrak{h}, \quad Q_{0}^{\mathrm{n}} \in \sum_{\alpha \in \Delta} \mathfrak{g}_{\alpha} .
$$

In that quadratic bundle case (9) is equivalent to the pair conditions given below:

$$
\lim _{|x| \rightarrow \infty} Q_{0}(x, t)=0, \quad \lim _{|x| \rightarrow \infty} Q_{1}(x, t)=0 .
$$

In order to formulate the direct scattering problem for $L(\lambda)$ one considers auxiliary linear system

$$
\mathrm{i} \partial_{x} \Psi(x, t, \lambda)+U(x, t, \lambda) \Psi(x, t, \lambda)=0
$$

where $\Psi$ is a fundamental set of solutions (or fundamental solution for short). Fundamental solutions take values in the Lie group G corresponding to $\mathfrak{g}$. We shall denote by $\mathcal{S}$ the space of all fundamental solutions to system (16). Let us introduce transform

$$
\Psi(x, t, \lambda) \quad \rightarrow \quad \tilde{\Psi}(x, t, \lambda)=g(x, t, \lambda) \Psi(x, t, \lambda)
$$


where $\Psi \in \mathcal{S}$ and $g$ takes values in the Lie group G. Transformations like (17) are known as gauge transformations [5]. Lax operators are transformed by the gauge transformation $g$ according to:

$$
\begin{aligned}
& L(\lambda) \rightarrow \tilde{L}(\lambda)=g L(\lambda) \hat{g}, \\
& A(\lambda) \rightarrow \tilde{A}(\lambda)=g A(\lambda) \hat{g}
\end{aligned}
$$

where the symbol ^ stands for the inverse of a matrix. Therefore the new operators $\tilde{L}(\lambda)$ and $\tilde{A}(\lambda)$ read

$$
\begin{aligned}
\tilde{L}(\lambda) & =\mathrm{i} \partial_{x}+\tilde{U}(x, t, \lambda), & & \tilde{U}=\mathrm{i} g \partial_{x} \hat{g}+g U \hat{g}, \\
\tilde{A}(\lambda) & =\mathrm{i} \partial_{t}+\tilde{V}(x, t, \lambda), & \tilde{V} & =\mathrm{i} g \partial_{t} \hat{g}+g V \hat{g} .
\end{aligned}
$$

It is immediately seen that the gauge transformations preserve the zero curvature condition (5), i.e. transformations $U \rightarrow \tilde{U}, \quad V \rightarrow \tilde{V}$ as given above are symmetries ${ }^{2}$ of equation (6). This is a manifestation of the fact that no one-to-one correspondence between Lax pairs and NLEs exists.

A very special example of gauge transformation playing an important role in theory of integrable systems, is dressing transform. In this case dressing matrix $g$ can be expressed in terms of a solution to the initial linear system which gives a quite effective way to derive another solution to a given NLE $[5,13]$.

Condition (5) implies any fundamental solution to (16) is also an eigenfunction of $A$ hence we have linear problem

$$
\mathrm{i} \partial_{t} \Psi+V \Psi=\Psi C
$$

for $C$ being some arbitrary function of $\lambda$. A special class of fundamental solutions are Jost solutions $\Psi_{+}$and $\Psi_{-}$determined by

$$
\lim _{x \rightarrow \pm \infty} \Psi_{ \pm}(x, t, \lambda) \mathrm{e}^{\mathrm{i} \mathcal{\partial}(\lambda) x}=\mathbb{1} .
$$

Definition (23) is correct provided

$$
C(\lambda)=\lim _{|x| \rightarrow \infty} V(x, t, \lambda) .
$$

That choice ensures the asymptotics of the Jost solutions are $t$-independent.

Transition matrix $T(t, \lambda)=\hat{\Psi}_{+}(x, t, \lambda) \Psi_{-}(x, t, \lambda)$ is called scattering matrix. It satisfies linear differential equation

$$
\mathrm{i} \partial_{t} T+[f(\lambda), T]=0
$$

where polynomial

$$
f(\lambda)=\lim _{|x| \rightarrow \infty} V(x, t, \lambda)
$$

is called dispersion law of NLE. Equation (25) represents a linearization of the original NLE and it can be easily integrated to give

$$
T(t, \lambda)=\mathrm{e}^{\mathrm{i} f(\lambda) t} T(0, \lambda) \mathrm{e}^{-\mathrm{i} f(\lambda) t} .
$$

\footnotetext{
${ }^{2}$ In general, symmetries do not necessarily respect boundary conditions, that is for a gauge transform $g$ to respect boundary condition it must be subject to some additional constraints.
} 
In what follows we shall impose simplifying condition $\mathcal{\partial}(\lambda)=\lambda^{M} J$ for $J \in \mathfrak{h}$ being real. The Jost solutions are defined for $\lambda^{M} \in \mathbb{R}$ only. It can be proven [3] there exist two fundamental solutions $X^{+}$and $X^{-}$analytic in domains

$$
\Omega^{+}=\left\{\lambda \in \mathbb{C}: \operatorname{Im} \lambda^{M} \geq 0\right\}, \quad \Omega^{-}=\left\{\lambda \in \mathbb{C}: \operatorname{Im} \lambda^{M} \leq 0\right\}
$$

respectively ${ }^{3}$. $X^{+}$and $X^{-}$are called fundamental analytic solutions and can be constructed in a purely algorithmic way from the Jost solutions using the following equalities:

$$
X^{ \pm}=\Psi_{-} S^{ \pm}=\Psi_{+} T^{\mp}
$$

where $S^{ \pm}$and $T^{ \pm}$appear in LU-decomposition

$$
T(t, \lambda)=T^{\mp}(t, \lambda) \hat{S}^{ \pm}(t, \lambda)
$$

of the scattering matrix, i.e. $S^{+}$and $T^{+}$are upper-triangular matrices while $S^{-}$and $T^{-}$are lower-triangular.

It is straightforward from $(27)$ that $X^{+}$and $X^{-}$are interrelated via

$$
X^{-}(x, \lambda)=X^{+}(x, \lambda) G(\lambda), \quad \operatorname{Im} \lambda^{M}=0 .
$$

In other words fundamental analytic solutions can be viewed as solutions to a local Riemann-Hilbert factorization problem [5, 13].

Fundamental analytic solutions allow one to describe the spectrum of the scattering operator. The resolvent of $L(\lambda)$ satisfies differential equation [5]

$$
L(\lambda) \circ R(\lambda)=\mathbb{1}
$$

It is an integral operator of the form:

$$
(R(\lambda) F)(x, t)=\int_{-\infty}^{\infty} \mathcal{R}(x, y, t, \lambda) F(y) \mathrm{d} y
$$

where $F: \mathbb{R} \rightarrow \mathbb{C}^{n}$ and $\mathcal{R}: \mathbb{R}^{3} \times \mathrm{D} \rightarrow \mathfrak{g}$ are assumed to be continuous in $y$ (and $x)$. The domain $\mathrm{D} \subset \mathbb{C}$ is complementary to the spectrum of the scattering operator, see $[3,5]$. The latter consists of two parts: a discrete part determined by the pole singularities of $\mathcal{R}$, if any, and a continuous part for which limit $\lim _{x, y \rightarrow \pm \infty} \mathcal{R}(x, y, t, \lambda)$ does not exist. It can be shown [3] that the integral kernel $\mathcal{R}$ can be expressed through the fundamental analytic solutions as given below:

$$
\mathcal{R}(x, y, \lambda)=\left\{\begin{aligned}
\mathrm{i} X^{+}(x, \lambda) \Theta^{+}(x-y) \hat{X}^{+}(y, \lambda), & \lambda \in \Omega^{+}, \\
-\mathrm{i} X^{-}(x, \lambda) \Theta^{-}(x-y) \hat{X}^{-}(y, \lambda), & \lambda \in \Omega^{-}
\end{aligned}\right.
$$

Functions $\Theta^{ \pm}(x-y)$ represent matrix Heavyside's step function, see [3] for more explanations. A more detailed analysis demonstrates that the continuous part of the spectrum of $L(\lambda)$ can be determined by condition $\operatorname{Im} \lambda^{M} J=0$, i.e. for polynomial bundles under consideration the continuous spectrum is a bunch of

\footnotetext{
${ }^{3}$ The cited paper by Gadzhiev, Gerdzhikov and Ivanov treats polynomial bundles associated with the Lie algebra $\mathfrak{s l}(2)$. However, the existence of fundamental analytic solutions and their construction in the case of arbitrary Lie algebra for real $J$ is quite similar to the case discussed in $[3]$.
} 
lines intersecting each other at the origin of the complex plane. Due to (31) $\mathcal{R}$ has singularities in $\lambda$ which are inherited from the corresponding singularities (or zeros) of $X^{+}$and $X^{-}$.

Let us see how gauge transformations affect the spectral properties of the scattering operator. We impose here natural requirement that $g$ respects the zero boundary condition (9), i.e. its asymptotics $g_{ \pm}=\lim _{x \rightarrow \pm \infty} g$ must be elements of the Cartan subgroup of the Lie group G. Then one notes that if fundamental solutions are transformed as given in (17), the resolvent is transformed according to

$$
R(\lambda) \rightarrow \tilde{R}(\lambda)=g R(\lambda) \hat{g}
$$

In the case of dressing transform (32) means that dressed resolvent acquires new singularities from the dressing matrix (and its inverse).

\section{Reductions}

Most $S$-integrable equations have Lax pairs with some additional algebraic symmetries. Those symmetries reduce the number of independent dynamical fields hence the name reductions $[5,13]$. A formalization of the notion of reduction is provided by Mikhailov's reduction group [8, 9].

Let $\mathrm{G}_{\mathrm{R}}$ be a finite group acting on the set of solutions $\mathcal{S}$. Suppose its action $\mathcal{K}: \mathrm{G}_{\mathrm{R}} \times \mathcal{S} \rightarrow \mathcal{S}$ is defined via:

$$
\mathcal{K}_{\mathrm{g}}: \Psi(x, t, \lambda) \rightarrow \tilde{\Psi}(x, t, \lambda)=\mathrm{K}_{\mathrm{g}}\left[\Psi\left(x, t, \hat{k}_{\mathrm{g}}(\lambda)\right)\right] \in \mathcal{S}, \quad \mathrm{g} \in \mathrm{G}_{\mathrm{R}}
$$

where $\Psi$ is an arbitrary fundamental solution, $k_{\mathrm{g}}: \mathbb{C} \rightarrow \mathbb{C}$ is a conformal mapping and $K_{\mathrm{g}} \in \operatorname{Aut}(\mathrm{G})$. As a result of (33) the Lax operators are transformed according to the rule:

$$
\begin{aligned}
& L(\lambda) \quad \rightarrow \quad \tilde{L}(\lambda)=\mathcal{K}_{\mathrm{g}} L(\lambda) \hat{\mathcal{K}}_{\mathrm{g}}, \\
& A(\lambda) \rightarrow \tilde{A}(\lambda)=\mathcal{K}_{\mathrm{g}} A(\lambda) \hat{\mathcal{K}}_{\mathrm{g}} .
\end{aligned}
$$

Due to the form of those transformation laws the zero curvature condition is preserved

$$
[\tilde{L}(\lambda), \tilde{A}(\lambda)]=\mathcal{K}_{\mathrm{g}}[L(\lambda), A(\lambda)] \hat{\mathcal{K}}_{\mathrm{g}}=0 .
$$

The invariance of the linear spectral problem implies that

$$
\tilde{L}(\lambda)=h(x, t, \lambda) L(\lambda)
$$

for some nondegenerate matrix $h(x, t, \lambda)$. Thus due to (36) and (37) the Lax pair acquires certain symmetries therefore they have fewer independent matrix elements. The group $\mathrm{G}_{\mathrm{R}}$ bears the name reduction group.

Example 3 Let us consider the following action of $\mathrm{G}_{\mathrm{R}}=\mathbb{Z}_{2}$

$$
\tilde{\Psi}(x, t, \lambda)=\hat{\Psi}^{\dagger}\left(x, t, \lambda^{*}\right),
$$

where operation $\dagger$ means performing matrix transposition and complex conjugation at the same time (that is Hermitian conjugation). As a result of the $\mathbb{Z}_{2}$ reduction (38) the Lax operators obey relations

$$
L^{\dagger}\left(\lambda^{*}\right)=L^{\mathrm{ad}}(\lambda), \quad A^{\dagger}\left(\lambda^{*}\right)=A^{\mathrm{ad}}(\lambda)
$$


where adjoint operator $L^{\mathrm{ad}}(\lambda)$ is defined as follows:

$$
L^{\mathrm{ad}}(\lambda) \Psi=-\mathrm{i} \partial_{x} \Psi+\Psi U .
$$

Therefore the functions $U$ and $V$ are subject to the following constraints

$$
U^{\dagger}\left(x, t, \lambda^{*}\right)=U(x, t, \lambda), \quad V^{\dagger}\left(x, t, \lambda^{*}\right)=V(x, t, \lambda) .
$$

For linear bundle Lax operators (see Example 1) constraint (40) leads to conclusion that $Q(x, t)$ is a Hermitian matrix, that is the number of independent fields is reduced twice.

The presence of reductions affects the spectral properties of the scattering operator. Indeed, taking into account relation (30) and the transformation law of $L(\lambda)$ we obtain that the resolvent transforms as follows:

$$
R(\lambda) \rightarrow \tilde{R}(\lambda)=\mathcal{K}_{g} R(\lambda) \hat{\mathcal{K}}_{g}=R(\lambda) \hat{h}(x, t, \lambda) .
$$

Relation (41) means that the spectrum of $L(\lambda)$ acquires symmetries, namely eigenvalues belong to orbits of the reduction group.

We shall discuss now how reductions affect gauge transformations. Suppose $g$ is a gauge transformation as given in (17). Taking into account (33) one can check that symmetry condition

$$
g=\mathcal{K}_{\mathrm{g}} g \hat{\mathcal{K}}_{\mathrm{g}} \quad \Rightarrow \quad \mathrm{K}_{\mathrm{g}}[g(x, t, \hat{k}(\lambda))]=g(x, t, \lambda)
$$

holds true. In particular, this means that dressing factor could not be arbitrary but should be symmetrized to fulfil condition (42). In Example 3 the dressing factor must satisfy constraint

$$
g^{\dagger}\left(x, t, \lambda^{*}\right)=\hat{g}(x, t, \lambda)
$$

This means that if $\lambda=\mu$ is a pole singularity of $g$ then $\lambda=\mu^{*}$ is a singularity of the inverse matrix $\hat{g}$. Thus both $\mu$ and $\mu^{*}$ could be poles ${ }^{4}$ of the dressed kernel $\mathcal{R}$ and thus contribute to the discrete spectrum of the dressed scattering operator.

Let us now consider a more general situation, namely we assume $G_{R}$ acts on the fundamental solutions by transforming independent variables as well

$$
\mathcal{K}_{\mathrm{g}}: \Psi(x, t, \lambda) \rightarrow \tilde{\Psi}(x, t, \lambda)=\mathrm{K}_{\mathrm{g}}\left[\Psi\left(\kappa_{\mathrm{g}}(x, t), k_{\mathrm{g}}(\lambda)\right)\right], \quad \mathrm{g} \in \mathrm{G}_{\mathrm{R}}
$$

where $k_{\mathrm{g}}$ and $\mathrm{K}_{\mathrm{g}}$ have the same meaning as before and $\kappa_{\mathrm{g}}: \mathbb{R}^{2} \rightarrow \mathbb{R}^{2}$ is a smooth mapping. Since the variables $x$ and $t$ are transformed as well we shall call this type of group action nonlocal reduction from now on. Let us list a few simple examples to illustrate that more general type of reduction.

Example 4 Ablowitz-Musslimani's reduction $\left(\mathrm{G}_{\mathrm{R}}=\mathbb{Z}_{2}\right)$

Let us consider a $\mathfrak{s l}(2)$-linear bundle, i.e. the scattering operator read:

$$
\begin{aligned}
L(\lambda) & =\mathrm{i} \partial_{x}+Q(x, t)-\lambda \sigma_{3}, \\
Q(x, t) & =\left(\begin{array}{cc}
0 & q(x, t) \\
p(x, t) & 0
\end{array}\right), \quad \sigma_{3}=\left(\begin{array}{cc}
1 & 0 \\
0 & -1
\end{array}\right) .
\end{aligned}
$$

${ }^{4}$ Whether or not the poles of the dressing factor and its inverse will also be poles of the dressed kernel $\mathcal{R}$, is determined by properties of the bare kernel $\mathcal{R}_{0}$, namely $\mathcal{R}_{0}$ must not have zeros at $\lambda=\mu$ and $\lambda=\mu^{*}$ to compensate those poles. 
Suppose $\mathbb{Z}_{2}$ acts on $\mathcal{S}$ in the following way:

$$
\sigma_{1} \Psi^{*}\left(-x, t,-\lambda^{*}\right) \sigma_{1}=\tilde{\Psi}(x, t, \lambda), \quad \sigma_{1}=\left(\begin{array}{cc}
0 & 1 \\
1 & 0
\end{array}\right) .
$$

Then the potential obeys symmetry condition

$$
\sigma_{1} Q^{*}(-x, t) \sigma_{1}=Q(x, t)
$$

and the functions $p$ and $q$ are interrelated via

$$
p(x, t)=q^{*}(-x, t) .
$$

The quadratic flow for the Lax operator (44) with condition (48) imposed yields to equation (1) with sign "+" before the cubic term, i.e. NNS "+" equation. To obtain NNS"-" one should impose the following $\mathbb{Z}_{2}$-action instead:

$$
\tilde{\Psi}(x, t, \lambda)=\hat{\Psi}^{\dagger}\left(-x, t,-\lambda^{*}\right) \quad \Rightarrow \quad Q^{\dagger}(-x, t)=-Q(x, t) .
$$

Hence one has:

$$
p(x, t)=-q^{*}(-x, t) .
$$

Example 5 Wadati's reduction $\left(\mathrm{G}_{\mathrm{R}}=\mathbb{Z}_{2} \times \mathbb{Z}_{2} \times \mathbb{Z}_{2}\right)$

Let us impose the following $\mathbb{Z}_{2}$ reductions on the operator (44)

$$
\hat{\Psi}^{\dagger}\left(x, t, \lambda^{*}\right)=\tilde{\Psi}(x, t, \lambda), \quad \hat{\Psi}^{T}(x, t,-\lambda)=\check{\Psi}(x, t, \lambda) .
$$

As a result of those reductions the potential acquires symmetries

$$
p(x, t)=q^{*}(x, t), \quad p(x, t)=-q(x, t),
$$

that is $q(x, t)=\mathrm{i} u(x, t)$ for $u: \mathbb{R}^{2} \rightarrow \mathbb{R}$. The cubic flow for $L$ with reductions (52) leads to the mKdV equation (2). Suppose now that in addition to (52) the following condition is imposed

$$
\sigma_{1} \Psi^{*}\left(-x,-t,-\lambda^{*}\right) \sigma_{1}=\breve{\Psi}(x, t, \lambda) .
$$

Then we immediately deduce that $u(-x,-t)=u(x, t)$, i.e. we have obtained the symmetry properties required by Wadati [11]. Similarly, if one requires

$$
\Psi^{*}\left(-x,-t, \lambda^{*}\right)=\bar{\Psi}(x, t, \lambda)
$$

instead of (53) then we have that $u(-x,-t)=-u(x, t)$. Thus reduction (53) (or (54)) leads to restriction of the manifold of all solutions to mKdV to the submanifold of even solutions (or odd solutions resp.).

The reduction effect on manifold of solutions as demonstrated in previous example motivates us to keep using the term reduction for the group action (43). To distinguish between (43) and the usual reduction action we shall refer to the former as nonlocal reduction while (33) will be called local reduction.

Example 6 Nonlocal Kaup-Newell's reduction $\left(\mathrm{G}_{\mathrm{R}}=\mathbb{Z}_{2} \times \mathbb{Z}_{2}\right)$

Consider $\mathfrak{s l}(2)$-quadratic bundle Lax operator

$$
L(\lambda)=\mathrm{i} \partial_{x}+\lambda\left[Q(x, t)-\lambda \sigma_{3}\right]
$$


where $Q$ and $\sigma_{3}$ are the same as in Example 4. Let us impose the following $\mathbb{Z}_{2}$ reduction:

$$
\hat{\Psi}^{T}(-x,-t, \lambda)=\tilde{\Psi}(x, t, \lambda) .
$$

Then we have

$$
Q^{T}(-x,-t)=Q(x, t) \quad \Leftrightarrow \quad p(x, t)=q(-x,-t) .
$$

The quadratic bundle (55) yields to an integrable hierarchy whose simplest nontrivial representative is nonlocal Kaup-Newell equation

$$
\mathrm{i} q_{t}+q_{x x}+\mathrm{i}\left[q^{2}(x, t) q(-x,-t)\right]_{x}=0 .
$$

Equation (58) as well as the original Kaup-Newell equation are PT-symmetric, i.e. they are invariant under transforms:

$$
t \rightarrow-t, \quad x \rightarrow-x, \quad q \rightarrow q^{*} .
$$

This type of reduction can be straightforwardly applied to a quadratic bundle related to any simple Lie algebra. For example, let $L(\lambda)$ be in the form:

$$
\begin{aligned}
L(\lambda) & =\mathrm{i} \partial_{x}+\lambda[Q(x, t)-\lambda J], \\
Q(x, t) & =\left(\begin{array}{cc}
0 & \mathbf{q}^{T}(x, t) \\
\mathbf{p}(x, t) & 0
\end{array}\right), \quad J=\left(\begin{array}{cc}
\mathbb{1}_{m} & 0 \\
0 & -\mathbb{1}_{n}
\end{array}\right)
\end{aligned}
$$

where $\mathbf{q}(x, t)$ and $\mathbf{p}(x, t)$ are $n \times m$-rectangular matrices. Then condition (57) means that

$$
\mathbf{p}(x, t)=\mathbf{q}(-x,-t) .
$$

This way equation (58) is generalized into

$$
\mathrm{i} \mathbf{q}_{t}+\mathbf{q}_{x x}+\mathrm{i}\left[\mathbf{q}(x, t) \mathbf{q}^{T}(-x,-t) \mathbf{q}(x, t)\right]_{x}=0
$$

which is a nonlocal counterpart of Fordy's multicomponent derivative nonlinear Schrödinger equations related to A.III symmetric spaces [2].

Like in the case of local reductions, the resolvent operator obeys condition (41) hence the spectrum of $L(\lambda)$ belongs to orbits of $G_{R}$. For example, the discrete eigenvalues of the linear bundle operator (44) appear in pairs $\left(\mu,-\mu^{*}\right)$, that is they are located symmetrically to the imaginary line (in particular, they can be purely imaginary).

Gauge transformations are affected by nonlocal reductions in a much the same way as in the case of local ones. The only difference is that the action of reduction group involves transformation of variables $x$ and $t$. More specifically, condition (42) still holds and it should be taken into account when picking up a dressing factor. For instance, when we have Ablowitz-Musslimani's reduction the dressing factor obeys the following symmetry condition [10]

$$
\sigma_{1} g^{*}\left(-x, t,-\lambda^{*}\right) \sigma_{1}=g(x, t, \lambda) .
$$

Similarly, in the case of Wadati's reduction (see Example 5) the dressing factor must satisfy the following set of relations:

$$
g^{\dagger}\left(x, t, \lambda^{*}\right)=\hat{g}(x, t, \lambda), \quad g^{T}(x, t,-\lambda)=\hat{g}(x, t, \lambda) .
$$


In addition to (64) $g$ must obey

$$
\sigma_{1} g^{*}\left(-x,-t,-\lambda^{*}\right) \sigma_{1}=g(x, t, \lambda)
$$

in the even solutions case or

$$
g^{*}\left(-x,-t, \lambda^{*}\right)=g(x, t, \lambda)
$$

in the odd solutions case respectively.

\section{Conclusions}

We have extended the original Mikhailov's notion of reduction group to reductions involving the independent variables as well, i.e. nonlocal type of reductions. We have demonstrated that the concept of nonlocal reductions can be successfully applied to describe nonlocal equations like NNS (1) or multicomponent derivative nonlinear Schrödinger equation (62). This substantially enlarges the class of NLEs which can be solved through the inverse scattering problem.

Not any discrete symmetry of the auxiliary spectral problem yield to reductions of a given NLE - it must respect the structure of the dispersion law of the NLE. For instance, Ablowitz-Musslimani's reduction (48) is compatible with NLEs whose dispersion law is a polynomial of even degree while Wadati's reduction (52), (53) is compatible with those of odd degree only.

The action of reduction group on independent variables provides us with a group-theoretical method to derive solutions with certain prescribed symmetries. The term reduction is appropriate here since one reduces the manifold of all solutions to a NLE onto the submanifold of those with the desired symmetries. If one applies the dressing method to obtain solutions to a NLE then the only thing they have to do is "symmetrize" the dressing factor so that it respects the reductions imposed, see formula (42) as well as (63)-(66). The symmetrization of the dressing factor here implies its singularities are not completely arbitrary but belong to certain orbits of the reduction group. Those singularities in turn give rise to singularities of the resolvent operator of $L(\lambda)$, see relation (32). Thus like in the classical "local case", the spectrum of the scattering operator is affected by reductions: it belongs to orbits of the reduction group.

We have considered in our paper polynomial bundle Lax operators. Most of the proposed constructions, however, can be applied to Lax operators of more general form like the rational bundle given below:

$$
L(\lambda)=\mathrm{i} \partial_{x}+U_{0}(x, t)+\sum_{k} \frac{U_{n_{k}}(x, t)}{\left(\lambda-a_{k}\right)^{n_{k}}}
$$

where $U_{0}(x, t)$ and $U_{n_{k}}(x, t)$ are elements of a simple Lie algebra. Those operators exhibit much richer properties compared to polynomial bundles. This is why their extensive study proves to be even more interesting but is technically much more involving. Due to all this it will be done elsewhere. 


\section{Acknowledgements}

The author would like to thank Dr. Rossen Ivanov for valuable discussions. Financial support from the Government of Ireland Postdoctoral Fellowship in Science, Engineering and Technology is also acknowledged.

\section{References}

[1] Ablowitz M. and Musslimani Z., Integrable Nonlocal Nonlinear Schrödinger Equation, Phys. Rev. Lett. 110, 064105(5) (2013).

[2] Fordy A., Derivative Nonlinear Schrödinger Equations and Hermitian Symmetric Spaces, J. Phys. A: Math. Gen. 17, 1235-1245 (1984).

[3] Gadzhiev I., Gerdzhikov V. and Ivanov M., Hamiltonian Structures of Nonlinear Evolution Equations Connected with a Polynomial Pencil, Zapiski Nauchnykh Seminarov LOMI 120, 55-68 (1982).

[4] Gerdjikov V. and Ivanov M., The Quadratic Bundle of General Form and the Nonlinear Evolution Equations. I. Expansions over the "Squared" Solitions - Generalized Fourier Transform, Bulg. J. Phys. 10, 13-26 (1983).

[5] Gerdjikov V., Vilasi G. and Yanovski A., Integrable Hamiltonian Hierarchies. Spectral and Geometric Methods, Lecture Notes in Physics $\mathbf{7 4 8}$ Springer Verlag, Berlin, Heidelberg, New York, 2008.

[6] Goto M. and Grosshans F., Semisimple Lie Algebras, Lecture Notes in Pure and Applied Mathematics 38 Marcel Dekker Inc., New York and Basel, 1978.

[7] Konopelchenko B., The Polynomial Spectral Problem of Arbitrary Order: A General Form of the Integrable Equations and Bäcklund Transformations, J. Phys. A: Math. Gen 14, 3125-3141 (1981).

[8] Mikhailov A., Reductions in Integrable Systems. The Reduction Groups, Lett. in J. of Exper. and Theor. Phys. 32, 187-192 (1980).

[9] Mikhailov A., The Reduction Problem and the Inverse Scattering Method, Physica D 3, 73-117 (1981).

[10] Valchev T., On a Nonlocal Nonlinear Schrödinger Equation, to appear in "Mathematics in Industry" Eds.: A. Slavova et al., Cambridge Scholars Publ., 2014 (Proc. of 8-th Annual Meeting of the Bulg. Section of SIAM, 18-19 December, 2013, Sofia, Bulgaria).

[11] Wadati M., Construction of Parity-time Symmetric Potential Through the Soliton Theory, J. Phys. Soc. Japan 77, 074005 (2008).

[12] Zakharov V. (Ed.), What is Integrability? Springer Series in Nonlinear Dynamics, Springer-Verlag, Berlin-Heidelberg-New York-London, 1990.

[13] Zakharov V., Manakov S., Novikov S. and Pitaevskii L., Theory of Solitons: The Inverse Scattering Method, Plenum, New York, 1984. 